\title{
Progress in High-Entropy Alloys
}

\author{
CHUAN ZHANG, ${ }^{1,4}$ MICHAEL C. GAO, ${ }^{2}$ and SHIH-KANG LIN $^{3}$ \\ 1.-Computherm, LLC, Middleton, WI 53562, USA. 2.-National Energy Technology Laboratory/ \\ Leidos Research Support Team, Albany, OR 97321, USA. 3.-Department of Materials Science \\ and Engineering, National Cheng Kung University, Tainan City 70101, Taiwan. \\ 4.—e-mail: Chuan.Zhang@computherm.com
}

High-entropy alloys (HEAs) loosely refer to multiprincipal-element solid-solution alloys because of their high configurational entropy. Their compositions are located near the center region of a multicomponent phase diagram, in contrast to traditional alloys that typically focus on the edge or corner of phase diagrams. The unique compositions and the resulting attractive properties of HEAs have stimulated ever-growing research interest because of scientific curiosity and potential industrial applications. The main objective of this special topic on high-entropy alloys is to collect contributions from the various fields of HEAs to disseminate the rapid progress in this fascinating and expanding class of advanced materials in a timely manner. In particular, we highlight the computational modeling, novel processing, mechanical properties, and oxidation behavior of HEAs.

Tong et al. review the recent experimental and theoretical progress on the local structure study with emphases on the local lattice distortion and charge transfer effect, which provides in-depth understandings of superb mechanical and radiation performances in strongly distorted HEAs. Artificial intelligence (AI) and machine learning (ML) are used in the design and development of HEAs. Agarwal et al. use an AI approach, an adaptiveneuro-fuzzy-interface system (ANFIS), to predict the formation of the BCC- and FCC-structured HEAs. The ANFIS is not only a tool but also a method for establishing a relation between parameters and phases in HEAs, which sheds light on understanding the phase formation in HEAs and represents a promising high-throughput approach for designing new HEAs. Chang et al. report the design of AlCoCrFeMnNi-based HEAs with the highest hardness by using the ML with the artificial

Shih-Kang Lin is the JOM advisor for the Alloy Phases Committee of the TMS Functional Materials and Structural Materials Divisions. Lin, Chuan Zhang, and Michael Gao were guest editors for the topic Progress in HighEntropy Alloys in this issue. neural network (ANN) method. The ML approach predicts three HEAs with hardness $>600 \mathrm{HV}$, and one HEA exhibits the highest hardness value among all alloys of the same HEA system.

The novel processing approaches to fabricating HEAs are explored. Peyrouzet et al. report the additive manufacture (AM) of the $\mathrm{Al}_{0.3} \mathrm{CoCrFeNi}$ HEA by powder-bed selective laser melting (SLM) and investigate its microstructure and tensile properties. The room temperature yield strength and ultimate tensile strength for as-printed SLM HEA are higher than those with as-cast and wrought counterparts while maintaining significant ductility. Nugroho et al. study the effects of WC addition on the friction-stir-processed (FSP) $\mathrm{Fe}_{50}(\mathrm{CoCrMnNi})_{50} \mathrm{HEAs}$. The formation of ultrafine grains in the FSP HEA leads to significant enhancement in the mechanical properties. Lu et al. systematically investigate the $\mathrm{FeCoCr}_{0.5} \mathrm{Al}_{x}$ HEAs prepared via the self-propagation high-temperature synthesis (SHS) method. The compressing yield strength, fracture strength, and fracture strain of the $\mathrm{FeCoNiCr}_{0.5} \mathrm{Al}_{0.8}$ SHS HEA are $1214 \mathrm{MPa}$, $2923 \mathrm{MPa}$, and $37 \%$, respectively.

The remaining five articles report new research results in the areas of thermal stability, mechanical property, and oxidation behavior of HEAs. Sadeghilaridjani et al. study the strain gradient plasticity of FCC single-phase HEAs in work-hardened and annealed conditions. Both series of alloys show higher hardness at shallower indentation depth attributed to geometrically necessary dislocations to accommodate the plastic deformation from the indenter geometry. Ding et al. investigate the effect of solid solution strengthening on the compressive strength of the $\mathrm{CoCrFeNiVAl}_{x}$ HEAs. It is found that high compressive strengths are due to the contribution of both solid solution strengthening and second-phase strengthening. Churyumov et al. report the microstructure and thermal and mechanical properties of the $(\mathrm{FeCoNiCuCr})_{100-x} \mathrm{Nb}_{x}$ HEAs in both as-cast and heat-treated states. The addition 
of $\mathrm{Nb}$ causes an increase in the yield strength by solid solution hardening and by the formation of intermetallic compounds. Jia et al. investigate the thermal stability and mechanical properties of a series of Ti-V-Ta alloys. These alloys show high thermal stability without the formation of secondary phases after annealing at $400-700^{\circ} \mathrm{C}$ for $48 \mathrm{~h}$ and exhibit good compression strength of 394$527 \mathrm{MPa}$ at $800^{\circ} \mathrm{C}$. Erdogan et al. study the effect of $\mathrm{Al}$ and $\mathrm{Ti}$ additions on the oxidation behavior of $\mathrm{CoCrFeNi}$-based HEAs. The formation of $\mathrm{Al}_{2} \mathrm{O}_{3}$ with the addition of $\mathrm{Al}$ due to the selective oxidation enhances the oxidation resistance of HEAs for better performance, while the presence of $\mathrm{Ti}$ has negatively affected the oxidation resistance of the HEAs.

The "Progress in High-Entropy Alloys" topic was proposed and supported by the Alloys Phases Committee (APC) of TMS for the October 2019 issue of $J O M$. Following is a list of the articles being published under this topic. To read or download any of the papers, follow the URL http://link.springe r.com/journal/11837/71/10/page/1 to the table of contents page for the October 2019 issue (vol. 71, no. $10)$.

- "Critical Review of Chemical Complexity Effect on Local Structure of Multi-principal Element Alloys" by Yang Tong and Fuxiang Zhang.

- "Artificial Intelligence Predicts Body-CenteredCubic and Face-Centered-Cubic Phases in HighEntropy Alloys" by Abhishek Agarwal and A.K. Prasada Rao.

- "Predictions of the Composition and Hardness for High-Entropy Alloys by Machine Learning" by Yao-Jen Chang, Chia-Yung Jui, Wen-Jay Lee, and An-Chou Yeh.

- "Selective Laser Melting of $\mathrm{Al}_{0.3} \mathrm{CoCrFeNi} H i g h-$ Entropy: Printability, Microstructure, and Mechanical Properties" by Florian Peyrouzet,
Dorian Hachet, R. Soulas, Christelle Navone, Stéphane Godet, and Stéphane Gorsse.

- "Effects of Tungsten-Carbide Particle Addition on Friction-Stir-Processed $\mathrm{Fe}_{50}(\mathrm{CoCrMnNi})_{50}$ Medium-Entropy Alloy" by Janu Ageng Nugroho, Dennis Edgard Jodi, Nokeun Park, Sungwook Kim, Unhae Lee, and Eung Ryul Baek.

- "FeCoNiCr ${ }_{0.5} \mathrm{Al}_{x}$ High-Entropy Alloys with DualPhase Solidification Microstructure and High Compressive Properties" by Tao Lu, Wenke Chai, Ting Dai, and Ye Pan.

- "Strain Gradient Plasticity in Multiprincipal Element Alloys" by Maryam Sadeghilaridjani and Sundeep Mukherjee.

- "Development of $\mathrm{CoCrFeNiVAl}_{x}$ High-Entropy Alloys Based on Solid Solution Strengthening" by Xiaolong Ding, Yongzhong Zhan, and Hongquan Tang.

- "Effect of $\mathrm{Nb}$ Addition on Microstructure and Thermal and Mechanical Properties of the Fe$\mathrm{Co}-\mathrm{Ni}-\mathrm{Cu}-\mathrm{Cr}$ Multiprincipal-Element (High-Entropy) Alloy in As-Cast and Heat-Treated State" by A.Yu. Churyumov, A.V. Pozdniakov, A.I. Bazlov, H. Mao, V.I. Polkin, and D.V. Louzguine-Luzgin.

- "Thermal Stability and Mechanical Properties of Low-Activation Single-Phase Ti-V-Ta Medium Entropy Alloys" by Nannan Jia, Yunkai Li, Xuan Liu, Yi Zheng, Benpeng Wang, Junsheng Wang, Yunfei Xue, and Ke Jin.

- "Effect of Al and Ti on High-Temperature Oxidation Behavior of CoCrFeNi-Based High-Entropy Alloys" by Azmi Erdogan, Kadir Mert Döleker, and Sakin Zeytin

Publisher's Note Springer Nature remains neutral with regard to jurisdictional claims in published maps and institutional affiliations. 